\title{
ON CERTAIN IDENTITIES IN THETA FUNCTIONS*
}

\author{
BY \\ CLAIBORNE G. LATIMER
}

1. The purpose of this paper is to obtain certain identities in Jacobi's theta functions by a new method. This method may be outlined as follows. We consider two sets of elements $S$ and $S_{1}$, conceptually distinct, such that the elements of $S$ are in one-to-one correspondence with the elements of $S_{1}$. In certain cases these elements are algebraic numbers and in other cases they are quaternions; in every case, corresponding elements have the same norms. We construct two functions $\Phi(x, y, \cdots)$ and $\Psi\left(x_{1}, y_{1}, \cdots\right)$, the terms of which are in one-to-one correspondence with the elements of $S$ and $S_{1}$ respectively. Hence there is a one-to-one correspondence between the terms of $\Phi$ and those of $\Psi$. We then show that if $x_{1}, y_{1}, \ldots$ are properly defined linear functions of $x, y, \cdots$, the corresponding terms are equal and therefore $\Phi(x, y, \cdots)=\Psi\left(x_{1}, y_{1}, \cdots\right)$.

We shall prove Jacobi's well known fundamental identity, $\dagger$ i.e.

$\Phi(x, y, z, w) \equiv \vartheta_{3}(x) \vartheta_{3}(y) \vartheta_{3}(z) \vartheta_{3}(w)+\vartheta_{2}(x) \vartheta_{2}(y) \vartheta_{2}(z) \vartheta_{2}(w)=\Phi\left(x_{1}, y_{1}, z_{1}, w_{1}\right)$,

where $x_{1}, y_{1}, z_{1}, w_{1}$ are certain linear functions of $x, y, z$, w. It will be shown that the terms of $\Phi$ are in one-to-one correspondence with the set of all (Hurwitz) integral quaternions. $\ddagger$ The above identity is then proved by employing certain well known properties of these quaternions. A generalization of this identity is obtained in a similar manner by employing sets of integral elements in generalized quaternion algebras. These sets were determined by the writer in a former paper, $\S$ employing a definition of integral element due to Dickson. $\uparrow$

The other identities are obtained by employing sets of elements not integral in general. We also obtain Schroeter's identity by the same method.

\footnotetext{
* Presented to the Society, March 30,1929, and December 27, 1929; received by the editors in October, 1929.

† Cf. Tannery and Molk, Eléments de la Théorie des Fonctions Elliptiques, vol. 2, p. 162. Kronecker's notation for the theta functions will be used throughout this paper.

$\ddagger$ Hurwitz, Vorlesungen über die Zahlentheorie der Quaternionen.

§ American Journal of Mathematics, vol. 48 (1926), pp. 57-66. We shall hereafter refer to this paper as J.

If Algebras and their Arithmetics, pp. 141, 2, 6. In J, Dickson's property $U^{\prime}$ was used instead of $U$. In the algebra of rational quaternions, the integral elements under the definition used in $J$ are the same as the Hurwitz integral elements.
} 
The use of algebraic numbers and quaternions, and in particular their arithmetics, to obtain identities in theta functions is believed to be new and not without interest.

2. Proof of Jacobi's fundamental identity. Part of the argument used to establish this identity may be repeated verbatim later on to prove a generalization.

We shall recall certain well known definitions and properties of real quaternions. If $X=x+y i+z j+w k$ is a real quaternion, where $i, j, k$ are the usual quaternion units, the conjugate of $X$ is $X^{\prime}=2 x-X$ and the norm of $X$ is $N(X)=X X^{\prime}=x^{2}+y^{2}+z^{2}+w^{2}$. Then $N(X Y)=N(X) N(Y)$ and $(X Y)^{\prime}=Y^{\prime} X^{\prime}$.

In the algebra of rational quaternions, the (Hurwitz) integral quaternions are those in the form

$$
\frac{1}{2}(\xi+\eta i+\zeta j+\lambda k)
$$

where $\xi, \eta, \zeta, \lambda$ are integers which are all even or all odd. The set $S$ of all such quaternions is closed under multiplication.

By definition*

$$
\begin{aligned}
& \vartheta_{2}(x, q)=\sum q^{(2 m+1)^{2} / 4} e^{(2 m+1) x i} \\
& \vartheta_{3}(x, q)=\sum q^{m^{2}} e^{2 m x i}
\end{aligned}
$$

where $q$ is a complex number, $|q|<1$. Since these functions are absolutely convergent we have, dropping the parameter $q$,

$$
\vartheta_{j}(x) \vartheta_{j}(y) \vartheta_{j}(z) \vartheta_{j}(w)=\sum q^{\left(\xi^{2}+\eta^{2}+\zeta^{2}+\lambda^{2}\right) / 4} e^{(\xi x+\eta y+\zeta z+\lambda w) i},
$$

where for $j=2[j=3]$ the summation extends over all systems of four odd [even] integers $\xi, \eta, \zeta, \lambda$. Assume for the present that $x, y, z, w$ are real and let

$$
\Omega=\frac{1}{2}(\xi+\eta i+\zeta j+\lambda k), \quad X=x+y i+z j+w k .
$$

Let the real part of the quaternion $Y$ be designated by $R(Y)$. Then

$$
\Phi(x, y, z, w)=\sum_{\Omega} q^{N(\Omega)} e^{2 i R\left(\Omega X^{\prime}\right)},
$$

where $\Phi$ is as defined in $\S 1$ and the summation extends, without duplication or omission, over all elements of $S$. The terms of $\Phi$ are thus in one-to-one correspondence with the elements of $S$.

Let $U$ be an element of $S$ of norm unity and let $x_{1}, y_{1}, z_{1}, w_{1}$ be defined in terms of $x, y, z, w$ by

$$
X_{1}=X U,
$$

* It will be understood throughout this paper that the limits of summation are $-\infty$ and $+\infty$ unless otherwise stated. 
where

$$
X_{1}=x_{1}+y_{1} i+z_{1} j+w_{1} k .
$$

Let $\Omega$ be an arbitrarily chosen element of $S$ and let $\tau$ be the corresponding term of $\Phi(x, y, z, w)$. Since $S$ is closed under multiplication,

$$
\Omega U=\Omega_{1}
$$

belongs to $S$. Let $\tau_{1}$ be the term of $\Phi\left(x_{1}, y_{1}, z_{1}, w_{1}\right)$ which corresponds to $\Omega_{1}$. We have

$$
\begin{aligned}
& N\left(\Omega_{1}\right)=N(\Omega) N(U)=N(\Omega), \\
& \Omega_{1} X_{1}^{\prime}=(\Omega U)(X U)^{\prime}=(\Omega U)\left(U^{\prime} X^{\prime}\right)=\Omega X^{\prime} .
\end{aligned}
$$

\section{Hence}

$$
\tau=q^{N(\Omega)} e^{2 i R\left(\Omega X^{\prime}\right)}=q^{N\left(\Omega_{1}\right)} e^{2 i R\left(\Omega_{1} X_{1}^{\prime}\right)}=\tau_{1} .
$$

Since $\tau=\tau_{1}$ is a formal identity in $x, y, z$, w, we may discard our assumption that they are real. $U$ belongs to $S$ and therefore the same is true of $U^{\prime}$. Hence by means of $\Omega U=\Omega_{1}$, we have a one-to-one correspondence between the terms of $\Phi(x, y, z, w)$ and $\Phi\left(x_{1}, y_{1}, z_{1}, w_{1}\right)$. And, as we have just seen, corresponding terms are equal. Therefore

$$
\Phi(x, y, z, w)=\Phi\left(x_{1}, y_{1}, z_{1}, w_{1}\right) .
$$

If we take as $U$ one of the elements

$$
\frac{1}{2}( \pm 1 \pm i \pm j \pm k)
$$

and change the signs of certain of the variables, (1) becomes Jacobi's identity. The only other elements in $S$ of norm unity are $\pm 1, \pm i, \pm j, \pm k$. If we take one of these as $U,(1)$ is trivial.

3. The sets of integral elements in generalized quaternion algebras. Consider the rational algebra $A$, with the basal numbers

$$
1, \quad I=(\beta \gamma)^{1 / 2} i, \quad J=(\gamma \alpha)^{1 / 2} j, \quad K=(\alpha \beta)^{1 / 2} k,
$$

where $\alpha, \beta, \gamma$ are positive odd integers such that $\alpha \beta \gamma$ contains no square factor $>1$. $A$ is a subalgebra of the algebra of real quaternions and hence has the properties mentioned in $\$ 2$.*

Two of $\alpha, \beta, \gamma$ are congruent, modulo 4, and therefore, after applying a cyclic substitution if necessary, we may assume that $\alpha \equiv \beta(\bmod 4)$. An integral element of $A$ is defined as an element which belongs to a set $S$ which

* In $\mathrm{J}$ we considered an algebra $A_{1}$, with a multiplication table which involved certain parameters $\alpha$ and $\beta$. Let $\alpha=\alpha_{1} \delta, \beta=\beta_{1} \delta$, where $\delta$ is the g.c.d. of $\alpha$ and $\beta$, and assume that $\alpha<0$ and $\beta>0$. If in the multiplication table of $A$.we replace $\alpha_{1}, \beta_{1}, \delta, K$ by $-\beta, \alpha, \gamma, \gamma K$ respectively, it will be seen that $A_{1}$ is equivalent to $A$. We shall quote the results of $\mathrm{J}$ in terms of the new notation. 
has certain properties $R, C, U, M *^{*}$ By property $C$, if $X, Y$ are elements of $S$ then $X Y$ also belongs to $S$. We shall not employ explicitly the properties $R, U, M$.

Let $\alpha=A \alpha^{\prime}, \beta=B \beta^{\prime}, \gamma=C \gamma^{\prime}$ where $A, B, C$ are defined as follows. $A$ is the product of all the prime factors $A_{i}$ of $\alpha$ such that $-\beta \gamma$ is a quadratic non-residue of every $A_{i}$; or $A=1$ if $\alpha$ contains no such factor. $B$ and $C$ are similarly defined with a cyclic substitution on $A, B, C$ and on $\alpha, \beta, \gamma$. If $Q$ is the number of prime factors in $\alpha^{\prime} \beta^{\prime} \gamma^{\prime}$, there are exactly $2^{Q}=t$ odd integers $H_{i}(i=1,2, \cdots, t)$, incongruent modulo $\alpha^{\prime} \beta^{\prime} \gamma^{\prime}$ such that

(2) $\beta \gamma H_{i}{ }^{2}+1 \equiv 0\left(\bmod \alpha^{\prime}\right), \gamma \alpha H_{i}{ }^{2}+1 \equiv 0\left(\bmod \beta^{\prime}\right), \alpha \beta H_{i}{ }^{2}+1 \equiv 0\left(\bmod \gamma^{\prime}\right)$.

Let $H$ be one of the $H_{i}$ and let $S_{i}\left[S_{i}{ }^{\prime}, S_{i}{ }^{\prime \prime}\right]$ be the totality of elements in the form

$$
\Omega=\frac{1}{2}\left(\xi+\frac{\eta}{\beta^{\prime} \gamma^{\prime}} I+\frac{\zeta}{\gamma^{\prime} \alpha^{\prime}} J+\frac{\lambda}{\alpha^{\prime} \beta^{\prime}} K\right),
$$

where $\xi, \eta, \zeta, \lambda$ are rational integers such that

$$
\begin{aligned}
\zeta \equiv B \gamma^{\prime} H \lambda & \left(\bmod \alpha^{\prime}\right) \\
\eta \equiv A \gamma^{\prime} H \lambda & \left(\bmod \beta^{\prime}\right), \\
\lambda \equiv A \beta^{\prime} H \zeta & \left(\bmod \gamma^{\prime}\right),
\end{aligned}
$$

and such that the first [second, third] of the following conditions is satisfied:

$$
\begin{aligned}
& \xi \equiv \eta \equiv \zeta \equiv \lambda, \\
& \xi \equiv \eta, \quad \zeta \equiv \lambda, \\
& \xi \equiv \zeta, \quad \eta \equiv \lambda
\end{aligned}
$$

We shall say that an element in the form (3) has the coördinates $\xi, \eta, \zeta, \lambda$. It will be noted that $S_{i}$ is the intersection of $S_{i}^{\prime}$ and $S_{i}^{\prime \prime}$.

If $\alpha \equiv \gamma(\bmod 4)$, by the proof of Theorem $3, \mathrm{~J}, S_{i}$ has the properties $R, C, U, M$. On the other hand, if $\alpha \equiv \gamma+2(\bmod 4)$, the sets $S_{i}^{\prime}$ and $S_{i}^{\prime \prime}$ have these properties. For every $H_{i}$ we thus have either one or two sets of integral elements.

From (2) it follows that (4) is equivalent to

$$
\begin{array}{rlrl}
\lambda & \equiv-\beta^{\prime} C H \zeta & & \left(\bmod \alpha^{\prime}\right), \\
\lambda \equiv-\alpha^{\prime} C H \eta & & \left(\bmod \beta^{\prime}\right), \\
\zeta \equiv-\alpha^{\prime} B H \eta & & \left(\bmod \gamma^{\prime}\right) .
\end{array}
$$

* J, p. 57. 
If $\eta, \zeta, \lambda$ satisfy (4) and if $\zeta \equiv \lambda$ or $\eta \equiv \lambda(\bmod 2)$, by employing (5) it may be shown that they may be written in the corresponding forms below:

(a)

$$
\begin{aligned}
& \eta=2 \beta^{\prime} \gamma^{\prime} m_{1}+r, \\
& \zeta=2 \gamma^{\prime} \alpha^{\prime} m_{2}-\alpha^{\prime} B H r+\beta \gamma^{\prime} H p, \\
& \lambda=2 \alpha^{\prime} \beta^{\prime} m_{3}-\alpha^{\prime} \mathrm{CHr}+\beta^{\prime} p \text {, } \\
& 0 \leqq p<2 \alpha^{\prime}, 0 \leqq r<2 \beta^{\prime} \gamma^{\prime} \text {; } \\
& \eta=2 \beta^{\prime} \gamma^{\prime} m_{1}+A \beta^{\prime} H r+\alpha \gamma^{\prime} H p, \\
& \eta \equiv \lambda(\bmod 2): \\
& \zeta=2 \gamma^{\prime} \alpha^{\prime} m_{2}+r \text {, } \\
& \lambda=2 \alpha^{\prime} \beta^{\prime} m_{3}-\beta^{\prime} C H r+\alpha^{\prime} p, \\
& 0 \leqq p<2 \beta^{\prime}, 0 \leqq r<2 \alpha^{\prime} \gamma^{\prime},
\end{aligned}
$$

where $m_{1}, m_{2}, m_{3}, r, p$ are integers, $r$ and $p$ subject to the indicated inequalities. Conversely, if $\eta, \zeta, \lambda$ may be written in the forms (6a) [(6b)], they satisfy $(4)$ and $\zeta \equiv \lambda[\eta \equiv \lambda](\bmod 2)$.

4. Construction of functions corresponding to the sets $S_{i}, S_{i}^{\prime}, S_{i}^{\prime \prime}$. Let $q$ be a complex number $|q|<1 ; a=A / \alpha^{\prime}, b=B / \beta^{\prime}, c=C / \gamma^{\prime}$, and

$$
\begin{aligned}
F_{1}(x, r) & =\sum_{m_{1}} q^{b c \eta^{2} / 4} e^{b c \eta x i} \\
G_{1}(x ; r, p) & =\sum_{m_{2}} q^{c a \zeta^{2} / 4} e^{c a \zeta x i} \\
H_{1}(x ; r, p) & =\sum_{m_{3}} q^{a b \lambda^{2} / 4} e^{a b \lambda x i}
\end{aligned}
$$

where $r, p$ are integers and $\eta, \zeta, \lambda$ are given by (6a). Let $F_{2}(x ; r, p), G_{2}(x, r)$, $H_{2}(x ; r, p)$ be defined in the same way except that we use the expressions (6b) for $\eta, \zeta, \lambda$. We may write

$$
F_{1}(x, r)=q^{b c r^{2} / 4} e^{b c r x i} \vartheta_{3}\left[B C(x-r k i), q^{\beta \gamma}\right],
$$

where $k=(\log q) / 2$. The other five functions may be similarly expressed in terms of theta functions. Hence each of these six functions is absolutely convergent. Then

$$
\begin{aligned}
\Psi_{0}(s, t) & =\vartheta_{3}(x) F_{1}(y, 2 s) G_{1}(z ; 2 s, 2 t) H_{1}(w ; 2 s, 2 t) \\
& =\sum q^{\left(\xi^{2}+b c \eta^{2}+c a \zeta^{2}+a b \lambda^{2}\right)} e^{(\xi x+b c \eta y+c a \zeta z+a b \lambda w) i},
\end{aligned}
$$

where the summation extends over all systems of four even integers $\xi, \eta, \zeta, \lambda$ such that $\eta, \zeta, \lambda$ may be written in the forms (6a) with $r, p$ replaced by $2 s, 2 t$ respectively. For every such system, there is a corresponding element of $S_{i}$,

$$
\Omega=\frac{1}{2}\left(\xi+\frac{\eta}{\beta^{\prime} \gamma^{\prime}} I+\frac{\zeta}{\gamma^{\prime} \alpha^{\prime}} J+\frac{\lambda}{\alpha^{\prime} \beta^{\prime}} K\right)
$$


Conversely, if $\Omega$ is an element of $S_{i}$ with even coördinates, $\xi, \eta, \zeta, \lambda$, there is a uniquely determined pair of integers $s, t, 0 \leqq s<\beta^{\prime} \gamma^{\prime}, 0 \leqq t<\alpha^{\prime}$, such that $\Psi_{0}(s, t)$ contains exactly one term corresponding to $\Omega$. Let

$$
\Psi_{1}(s, t) \equiv \vartheta_{2}(x) F_{1}(y, 2 s+1) G_{1}(z ; 2 s+1,2 t) H_{1}(w ; 2 s+1,2 t) .
$$

The above argument regarding $\Psi_{0}$ may be repeated for $\Psi_{1}$ except that $\xi, \eta, \zeta, \lambda$ are odd, $r$ is replaced by $2 s+1$, and $\Omega$ has odd coördinates. Hence

$$
\begin{aligned}
\Phi_{1}(x, y, z, w) & \equiv \sum_{\boldsymbol{s}=0}^{\beta^{\prime} \gamma^{\prime}-1} \sum_{t=0}^{\alpha^{\prime}-1}\left(\Psi_{0}(s, t)+\Psi_{1}(s, t)\right) \\
& =\sum_{\mathbf{\Omega}} q^{N(\boldsymbol{\Omega})} e^{2 R\left(\boldsymbol{\Omega} \mathbf{X}^{\prime}\right) i},
\end{aligned}
$$

where we assume for the present that $x, y, z, w$ are real,

$$
X=x+\frac{y}{\beta^{\prime} \gamma^{\prime}} I+\frac{z}{\gamma^{\prime} \alpha^{\prime}} J+\frac{w}{\alpha^{\prime} \beta^{\prime}} K,
$$

and the summation extends, without duplication or omission, over all elements of $S_{i}$. The terms of $\Phi_{1}$ are thus in one-to-one correspondence with the elements of $S_{i}$.

We shall next construct functions $\Phi_{2}$ and $\Phi_{3}$, the terms of which are in one-to-one correspondence with the elements of $S_{i}^{\prime}$ and $S_{i}^{\prime \prime}$ respectively. As noted before, both $S_{i}^{\prime}$ and $S_{i}^{\prime \prime}$ contain $S_{i}$. Accordingly, we shall obtain $\Phi_{2}$ and $\Phi_{3}$ by adding properly chosen functions to $\Phi_{1}$.

The $\eta, \zeta, \lambda$ coördinates of every element of $S_{\boldsymbol{i}}^{\prime}$ may be written in the forms (6a) and $\xi \equiv \eta, \zeta \equiv \lambda(\bmod 2)$. Furthermore, if such an element does not belong to $S_{i}, \eta \equiv \lambda+1(\bmod 2)$, and therefore in $(6 a) r \equiv p+1(\bmod 2)$. Therefore if we set

$$
\begin{aligned}
& \phi_{20} \equiv \vartheta_{3}(x) \sum_{s=0}^{\beta^{\prime} \gamma^{\prime}-1} \sum_{t=0}^{\alpha^{\prime}-1} F_{1}(y, 2 s) G_{1}(z ; 2 s, 2 t+1) H_{1}(w ; 2 s, 2 t+1), \\
& \phi_{21} \equiv \vartheta_{2}(x) \sum_{s=0}^{\beta^{\prime} \gamma^{\prime}-1} \sum_{t=0}^{\alpha^{\prime}-1} F_{1}(y, 2 s+1) G_{1}(z ; 2 s+1,2 t+1) H_{1}(w ; 2 s+1,2 t+1),
\end{aligned}
$$

it follows that $\phi_{20}+\phi_{21}$ is equal to the expression on the right of (7), where in this case the summation extends over all the elements of $S_{i}^{\prime}$ which do not belong to $S_{i}$. Then

$$
\Phi_{2}(x, y, z, w) \equiv \Phi_{1}+\phi_{20}+\phi_{21}=\sum_{\Omega} q^{N(\boldsymbol{Q})} e^{2 i R\left(\mathbf{\Omega} \mathbf{X}^{\prime}\right)},
$$

where the summation extends over all the elements of $S_{\boldsymbol{i}}^{\prime}$. If we let 


$$
\begin{aligned}
& \phi_{30} \equiv \vartheta_{3}(x) \sum_{s=0}^{\gamma^{\prime} \alpha^{\prime-1}} \sum_{t=0}^{\beta^{\prime}-1} F_{2}(y ; 2 s, 2 t+1) G_{2}(z, 2 s) H_{2}(w ; 2 s, 2 t+1), \\
& \phi_{31} \equiv \vartheta_{2}(x) \sum_{s=0}^{\gamma^{\prime} \alpha^{\prime-1}} \sum_{t=0}^{\beta^{\prime}-1} F_{2}(y ; 2 s+1,2 t+1) G_{2}(z, 2 s+1) H_{2}(w ; 2 s+1,2 t+1),
\end{aligned}
$$

it may be shown as for $\Phi_{2}$ that

$$
\Phi_{3}(x, y, z, w) \equiv \Phi_{1}+\phi_{30}+\phi_{31}=\sum_{\Omega} q^{N(\Omega)} e^{2 i R\left(\Omega X^{\prime}\right)},
$$

where now the summation extends over all the elements of $S_{i}^{\prime \prime}$.

5. 'Identities in functions which correspond to sets of integral elements. For each of the sets $S_{i}, S_{i}^{\prime}, S_{i}^{\prime \prime}$ we have a corresponding function $\Phi_{j}(j=1,2,3)$. As noted in $\S 3, S_{i}$ has the properties $R, C, U, M$ if $\alpha \equiv \gamma(\bmod 4)$, while $S^{\prime}$ and $S^{\prime \prime}$ have these properties if $\alpha \equiv \gamma+2(\bmod 4)$. Let $S$ be one of the sets which has these properties and let $\Phi$ be the corresponding function. Let

$$
X_{1}=x_{1}+\frac{y_{1}}{\beta^{\prime} \gamma^{\prime}} I+\frac{z_{1}}{\gamma^{\prime} \alpha^{\prime}} J+\frac{w_{1}}{\alpha^{\prime} \beta^{\prime}} K \text {. }
$$

The argument of $\$ 2$ beginning "Let $U$ be an element of $S \ldots$ " and leading to (1) may now be repeated word for word, it being understood that we employ the $\Phi, X_{1}$ and $S$ of this paragraph and the $X$ and $\Omega$ of the preceding paragraph. We have then

THEOREM 1. Let $S$ be one of the sets $S_{i}, S_{i}^{\prime}, S_{i}^{\prime \prime}$ which has the properties $R, C, U, M$, and let $\Phi$ be the corresponding $\Phi_{j}$. If $U$ is an element of $S$ of norm unity and if $x_{1}, y_{1}, z_{1}, w_{1}$ are defined in terms of $x, y, z$, w by means of $X U=X_{1}$, then*

$$
\Phi(x, y, z, w)=\Phi\left(x_{1}, y_{1}, z_{1}, w_{1}\right) .
$$

6. Special cases of Theorem 1. If $\alpha, \beta, \gamma$ are assigned numerical values, to write out explicitly the identity of the above theorem it is necessary to determine an element $U$ as there defined, i.e. a unit belonging to $S$. In some cases a unit yields a trivial identity, as was seen at the end of $\$ 2$. Furthermore, the algebra may have no unit which yields a non-trivial identity. This is the case if $\alpha=\beta=1, \gamma=7$; the only units being $\pm 1, \pm K$.

By the proofs of Theorems 2 and 3 of $\mathrm{J}$, every element with integral coördinates and integral norm belongs to a set of integral elements. The

* We have considered only those algebras in which $\alpha, \beta, \gamma$ are odd. The same method is being used in a Kentucky thesis, employing the algebras in which one of these parameters is even and the results of Professor Darkow on the arithmetics of such algebras. See Annals of Mathematics, vol. 28, pp. 263-70. 
problem of determining those algebras which contain units yielding nontrivial identities is therefore equivalent to that of determining those values of $\alpha, \beta, \gamma$ such that the equation

$$
\alpha^{\prime} \beta^{\prime} \gamma^{\prime} \xi^{2}+\alpha^{\prime} B C \eta^{2}+\beta^{\prime} C A \zeta^{2}+\gamma^{\prime} A B \lambda^{2}=4 \alpha^{\prime} \beta^{\prime} \gamma^{\prime}
$$

has a solution in integers, two of which are $\neq 0$. This, of course, is a very difficult problem. We shall give two classes of algebras in which such a unit may be determined and the corresponding identity written out explicitly.

Let $\beta=\gamma=1$ and let $\alpha$ be a product of distinct primes, each in the form $4 n+1$. Then $A=B=C=\beta^{\prime}=\gamma^{\prime}=1, \alpha=\alpha^{\prime}=u^{2}+v^{2}$, where $u, v$ are relatively prime. We may take

$$
U=\frac{1}{2}\left(1+I+\frac{u+v}{\alpha} J+\frac{u-v}{\alpha} K\right) .
$$

For example, if $\alpha=5, u=2, v=1, H=3$, it will be found that $\Phi$ may be written

$$
\begin{aligned}
\Phi(x, y, z, w) & =\vartheta_{3}(x, q) \vartheta_{3}(y, q) \sum_{t=0}^{4} q^{2 t^{2}} e^{2(3 z+w) t i / 5} \vartheta_{3}\left(z-6 t k i, q^{5}\right) \vartheta_{3}\left(w-2 t k i, q^{5}\right) \\
& +\vartheta_{2}(x, q) \vartheta_{2}(y, q) \sum_{t=0}^{4} q^{2 t^{2}} e^{2(3 z+w) t i / 5} \vartheta_{2}\left(z-6 t k i, q^{5}\right) \vartheta_{2}\left(w-2 t k i, q^{5}\right) .
\end{aligned}
$$

By the theorem,

where we find

$$
\Phi(x, y, z, w)=\Phi\left(x_{1}, y_{1}, z_{1}, w_{1}\right)
$$

$$
\begin{array}{ll}
x_{1}=\frac{1}{2}\left(x-y-\frac{3 z+w}{5}\right), & z_{1}=\frac{1}{2}(3 x-y+z+w), \\
y_{1}=\frac{1}{2}\left(x+y+\frac{z-3 w}{5}\right), & w_{1}=\frac{1}{2}(x+3 y-z+w) .
\end{array}
$$

Similar results may be obtained if $\alpha$ is a product of distinct primes, each in the form $3 n+1$, one of $\beta, \gamma$ is unity and the other is 3 , noting that the notation must be so chosen that $\alpha \equiv \beta(\bmod 4)$.

If the method of the preceding paragraphs be applied to the algebraic integers in the field defined by an imaginary cube root of unity and if we set

$$
\Phi(x, y) \equiv \vartheta_{3}(x, q) \vartheta_{3}\left(y, q^{3}\right)+\vartheta_{2}(x, q) \vartheta_{2}\left(y, q^{3}\right),
$$

we obtain the known identity

$$
\Phi(x, y)=\Phi\left(x_{1}, y_{1}\right),
$$


where

$$
x_{1}=\frac{1}{2}(x+y), \quad y_{1}=\frac{1}{2}(3 x-y) \text {. }
$$

7. Proof of Schroeter's identity. The sets of elements used hereafter will not be integral in general. Let $r, p$ be positive integers and let $s, t$ be integers such that $t$ is prime to

$$
\Delta \equiv r s^{2}+p t^{2} .
$$

Let $\rho=r^{1 / 2}, \tau=i p^{1 / 2}$, and let $S$ be the totality of numbers in the form

$$
\Omega=\Delta^{-1 / 2}(\xi+\eta \rho \tau),
$$

where $\xi, \eta$ are integers such that

$$
t \xi \equiv-r s \eta
$$

Let $S_{1}$ be the the totality of numbers in the form

$$
\Omega_{1}=\xi_{1} \rho+\eta_{1} \tau,
$$

where $\xi_{1}, \eta_{1}$ are integers. If $U \equiv \Delta^{-1 / 2}(s \rho+t \tau)$ and

$$
\Omega U=\Omega_{1},
$$

it may be shown that $\Omega_{1}$ belongs to $S_{1}$ if and only if $\Omega$ belongs to $S$. The numbers of $S$ are therefore in one-to-one correspondence with the numbers of $S_{1}$.

It may be shown that if $\mu$ is an integer and if

$$
\Phi_{\mu}(x, y) \equiv q^{r \mu^{2}} e^{2 r(s x-p t y) \mu i} \vartheta_{3}\left(\Delta x-r s \mu k i, q^{\Delta}\right) \vartheta_{3}\left(p r \Delta y+p r t \mu k i, q^{p r \Delta}\right),
$$

where $k=\log q$, then

$$
\Phi_{\mu}(x, y)=\sum q^{\left(\xi^{2}+p r \eta^{2}\right) / \Delta} e^{2(\xi x+p r \eta y) i},
$$

where the summation extends over all pairs of integers $\xi, \eta$ such that

Then

$$
\xi \equiv r s \mu, \quad \eta \equiv-t \mu
$$

where

$$
\Phi(x, y) \equiv \sum_{\mu=0}^{\Delta-1} \Phi_{\mu}(x, y)=\sum_{\Omega} q^{N(\Omega)} e^{2 i R\left(\Omega X^{\prime}\right)}
$$

$$
X=\Delta^{1 / 2}(x+y \rho \tau)
$$

and the summation extends over all the numbers of $S$.

It is readily shown that

where

$$
\Psi\left(x_{1}, y_{1}\right) \equiv \vartheta_{3}\left(r x_{1}, q^{r}\right) \vartheta_{3}\left(p y_{1}, q^{p}\right)=\sum_{\Omega_{1}} q^{N\left(\Omega_{1}\right)} e^{2 i R\left(\Omega_{1} X_{1}^{\prime}\right)}
$$

$$
X_{1}=x_{1} \rho+y_{1} \tau
$$


and the summation extends over all the numbers of $S_{1}$.

Let $x_{1}, y_{1}$ be defined in terms of $x, y$ by

$$
X_{1}=X U \text {. }
$$

By means of $\Omega_{1}=\Omega U$ we have a one-to-one correspondence between the terms of $\Phi$ and $\Psi$. For corresponding terms,

$$
\begin{aligned}
& N\left(\Omega_{1}\right)=N(\Omega) N(U)=N(\Omega), \\
& \Omega_{1} X_{1}^{\prime}=(\Omega U)\left(U^{\prime} X^{\prime}\right)=\Omega X^{\prime} .
\end{aligned}
$$

Hence corresponding terms are equal and

$$
\Phi(x, y)=\Psi\left(x_{1}, y_{1}\right) .
$$

If this is expressed in terms of theta functions and $y$ is replaced by $-y / p r$, we obtain Schroeter's formula:

$$
\begin{aligned}
\vartheta_{3}\left(r s x+t y, q^{r}\right) \vartheta_{3}( & \left.-p t x+s y, q^{p}\right) \\
& =\sum_{\mu=0}^{\Delta-1} q^{r \mu^{2}} e^{2(r s x+t y) \mu i} \vartheta_{3}\left(\Delta x-r s \mu k i, q^{\Delta}\right) \vartheta_{3}\left(\Delta y-p r t \mu k i, q^{p r \Delta}\right) .
\end{aligned}
$$

8. An analogue of Schroeter's identity. Let $p, r, s, t$ be defined as in the preceding paragraph, except that

$$
r s^{2}+p t^{2}=\Delta^{2},
$$

where $\Delta$ is a positive integer. Let $S\left[S_{1}\right]$ be the totality of numbers in the form

$$
\Omega=\xi+\eta \rho \tau \quad\left[\Omega_{1}=\xi_{1} \rho+\eta_{1} \tau\right],
$$

where $\xi, \eta\left[\xi_{1}, \eta_{1}\right]$ are integers such that

$$
t \xi \equiv-r s \eta \quad\left[t \xi_{1} \equiv s \eta_{1}\right]
$$

Let $U=s \rho+t r$. If

$$
\Omega U=\Delta \Omega_{1},
$$

it may be shown that $\Omega_{1}$ belongs to $S_{1}$ if and only if $\Omega$ belongs to $S$. Let $\mu$ be an arbitrarily chosen integer and

$$
\Phi_{\mu}(x, y) \equiv q^{r \mu^{2} \Delta^{2}} e^{2 r(s x-p t y) \mu i} \vartheta_{3}\left(\Delta x-r s \mu \Delta k i, q^{\Delta^{2}}\right) \vartheta_{3}\left(p r \Delta y+p r t \mu \Delta k i, q^{p r \Delta^{2}}\right) .
$$

Then

$$
\Phi_{\mu}(x, y)=\sum q^{\xi^{2}+p r \eta^{2}} e^{2(\xi x+p r \eta y) i},
$$

where the summation extends over all pairs of integers $\xi, \eta$ such that

$$
\xi \equiv r s \mu, \quad \eta \equiv-t \mu
$$


Then

$$
\Phi(x, y) \equiv \sum_{\mu=0}^{\Delta-1} \Phi_{\mu}(x, y)=\sum_{\mathbf{\Omega}} q^{N(\Omega)} e^{2 i R\left(\Omega X^{\prime}\right)},
$$

where the summation extends over all the numbers of $S$ and $X=x+y \rho \tau$.

If we set

$\Psi\left(x_{1}, y_{1}\right) \equiv \sum_{\mu=0}^{\Delta-1} q^{\Delta^{2} \mu^{2}} e^{2 \mu\left(r r x_{1}+p t y_{1}\right) i} \vartheta_{3}\left(r \Delta x_{1}-r s \mu \Delta k i, q^{r \Delta^{2}}\right) \vartheta_{3}\left(p \Delta y_{1}-p t \mu \Delta k i, q^{p \Delta^{2}}\right)$,

it may be shown in a similar manner that

$$
\Psi\left(x_{1}, y_{1}\right)=\sum_{\mathbf{\Omega}_{1}} q^{N\left(\Omega_{1}\right)} e^{2 i R\left(\Omega_{1} X_{1}{ }^{\prime}\right)},
$$

where the summation extends over all the numbers of $S_{1}$ and $X_{1}=x_{1} \rho+y_{1} \tau$.

By (9), the terms of $\Phi$ are in one-to-one correspondence with the terms of $\Psi$; and furthermore, for corresponding terms, $N(\Omega)=N\left(\Omega_{1}\right)$. Hence if we set

it follows that

$$
\Delta X_{1}=X U
$$

$$
\Phi(x, y)=\Psi\left(x_{1}, y_{1}\right) .
$$

If we express this in terms of theta functions and replace $q^{\Delta^{2}}, \Delta x, \operatorname{pr} \Delta y, r \Delta x_{1}$, $p \Delta y_{1}$ by $q, x, y, x_{1}, y_{1}$ respectively, we obtain the identity below.

Theorem 2. If $p, r$ are positive integers and $s, t, u, v$ are integers such that

$$
r s^{2}+p t^{2}=\Delta^{2},
$$

where $\Delta$ is a positive integer prime to $t$, then

where

$$
\begin{aligned}
& \sum_{\mu=0}^{\Delta-1} q^{r \mu^{2}} e^{2 \mu x_{1} i} \vartheta_{3}\left(x-\frac{r s \mu k i}{\Delta}, q\right) \vartheta_{3}\left(y+\frac{p r t \mu k i}{\Delta}, q^{p r}\right) \\
= & \sum_{\mu=0}^{\Delta-1} q^{\mu^{2}} e^{2 \mu x i} \vartheta_{3}\left(x_{1}-\frac{r s \mu k i}{\Delta}, q^{r}\right) \vartheta_{3}\left(y_{1}-\frac{p t \mu k i}{\Delta}, q^{p}\right),
\end{aligned}
$$

$$
x_{1}=(r s x-t y) / \Delta, \quad y_{1}=(p t x+s y) / \Delta .
$$

For the case $r=s=t=1, p=3$, the identity of the above theorem is equivalent to the identity mentioned at the end of $\S 6$. For $r=p=2, s=t=1$, the above identity reduces to

$$
\begin{gathered}
\vartheta_{3}(x, q) \vartheta_{3}(y, q)=\vartheta_{3}\left(x_{1}, q^{2}\right) \vartheta_{3}\left(y_{1}, q^{2}\right)+\vartheta_{2}\left(x_{1}, q^{2}\right) \vartheta_{2}\left(y_{1}, q^{2}\right), \\
x_{1}=x-y, \quad y_{1}=x+y .
\end{gathered}
$$

This is a special case of Schroeter's identity. 
9. An extension of Schroeter's identity. In this and the next paragraph we shall obtain identities in functions of four variables similar to Schroeter's identity and to the identity of the preceding paragraph.

Let $a, b, c$ be positive integers and let $s, t, u, v$ be integers such that

$$
s^{2}+b c t^{2}+c a u^{2}+a b v^{2} \equiv \Delta
$$

is prime to $\delta \equiv s^{2}+b c t^{2}$. Let

$$
I=(b c)^{1 / 2} i, J=(c a)^{1 / 2} j, K=(a b)^{1 / 2} k,
$$

where $i, j, k$ are the quaternion units, and let

$$
A=s u-b t v, \quad B=s v+c t u .
$$

Let $S$ be the totality of quaternions in the form

$$
\Omega=\Delta^{-1 / 2}(\xi+\eta I+\zeta J+\lambda K),
$$

where $\xi, \eta, \zeta, \lambda$ are integers such that

$$
\begin{aligned}
& \delta \xi \equiv a c A \zeta+a b B \lambda, \\
& \delta \eta \equiv-a B \zeta+a A \lambda
\end{aligned}
$$

$(\bmod \Delta)$

Let $S_{1}$ be the totality of quaternions in the form

$$
\Omega_{1}=\xi_{1}+\eta_{1} I+\zeta_{1} J+\lambda_{1} K,
$$

where $\xi_{1}, \eta_{1}, \zeta_{1}, \lambda_{1}$ are integers, and let

$$
U=\Delta^{-1 / 2}(s+t !+u J+v K) .
$$

It may be shown that if

$$
\Omega U=\Omega_{1},
$$

then $\Omega_{1}$ belongs to $S_{1}$ if and only if $\Omega$ belongs to $S$. We have therefore by means of (11) a one-to-one correspondence between the elements of $S$ and those of $S_{1}$.

If $\xi, \eta, \zeta, \lambda$ satisfy (10), there is a uniquely determined pair of integers $\mu, \nu ; 0 \leqq \mu<\Delta, 0 \leqq \nu<\Delta$, such that

$$
\xi \equiv \delta \mu, \quad \eta \equiv \delta \nu
$$

By means of (10) we find

$$
\zeta \equiv-A \mu+b B \nu, \quad \lambda \equiv-(B \mu+c A \nu) \quad(\bmod \Delta) .
$$

Conversely, if $\mu, \nu$ are arbitrarily chosen integers and if $\xi, \eta, \zeta, \lambda$ satisfy (12) and (13), they also satisfy (10). Let 


$$
\begin{gathered}
\rho=-A \mu+b B \nu, \quad \tau=-(B \mu+c A \nu), \\
\Phi_{\mu, \nu} \equiv q^{\delta\left(\mu^{2}+b c \nu^{2}\right)} e^{2(\delta \mu x+b c \delta \nu \nu+c a \rho z+a b \tau w) i} \vartheta_{3}\left(\Delta x-\delta \mu k i, q^{\Delta}\right) \\
\cdot \vartheta_{3}\left[b c(\Delta y-\delta \nu k i), q^{b c \Delta}\right] \vartheta_{3}\left[c a(\Delta z-\rho k i), q^{c a \Delta}\right] \vartheta_{3}\left[a b(\Delta w-\tau k i), q^{a b \Delta}\right]
\end{gathered}
$$

where $k=\log q$. It may be shown that

$$
\Phi_{\mu \nu}=\sum q^{\left(\xi^{2}+b c \eta^{2}+a c \zeta^{2}+a b \lambda^{2}\right) / \Delta} e^{2\left(\xi x+b c \eta y+c a \xi_{z}+a b \lambda w\right) i}
$$

where the summation extends over all quadruples $\xi, \eta, \zeta, \lambda$ which satisfy (12) and (13).

If we set $X=\Delta^{1 / 2}(x+y I+z J+w K)$ it follows that

$$
\Phi(x, y, z, w) \equiv \sum_{\mu, \nu=0}^{\Delta-1} \Phi_{\mu \nu}=\sum_{\Omega} q^{N(\Omega)} e^{2 i R\left(\Omega X^{\prime}\right)},
$$

where the summation extends over all the elements of $S$. It is readily shown that if

$$
\Psi\left(x_{1}, y_{1}, z_{1}, w_{1}\right) \equiv \vartheta_{3}\left(x_{1}, q\right) \vartheta_{3}\left(b c y_{1}, q^{b c}\right) \vartheta_{3}\left(c a z_{1}, q^{c a}\right) \vartheta_{3}\left(a b w_{1}, q^{a b}\right),
$$

then

$$
\Psi\left(x_{1}, y_{1}, z_{1}, w_{1}\right)=\sum_{\Omega_{1}} q^{N\left(\Omega_{1}\right)} e^{\dot{2 i R\left(\Omega_{1} X_{1}{ }^{\prime}\right)},}
$$

where the summation extends over all the elements of $S_{1}$ and $X_{1}=x_{1}+y_{1} I$ $+z_{1} J+w_{1} K$. By means of (11) there is a one-to-one correspondence between the terms of $\Phi$ and those of $\Psi$. If we let $x_{1}, y_{1}, z_{1}, w_{1}$ be defined in terms of $x, y, z, w$ by $X_{1}=X U$, it may be shown as in the preceding proofs that the corresponding terms of $\Phi$ and $\Psi$ are equal and hence

$$
\Phi(x, y, z, w)=\Psi\left(x_{1}, y_{1}, z_{1}, w_{1}\right) .
$$

Expressing this in terms of theta functions, replacing $y, z, w$ by $y /(b c)$, $z /(c a), w /(a b)$ respectively, and making similar replacements for $y_{1}, z_{1}, w_{1}$, we obtain the identity below.

TheOREM 3. Let $a, b, c$ be positive integers and let $s, t, u, v$ be integers such that

$$
s^{2}+b c t^{2}+c a u^{2}+a b v^{2} \equiv \Delta
$$

is prime to $\delta=s^{2}+b c t^{2}$. Let $A=s u-b t v, B=s v+c t u$. Then

$$
\begin{gathered}
\vartheta_{3}\left(x_{1}, q\right) \vartheta_{3}\left(y_{1}, q^{b c}\right) \vartheta_{3}\left(z_{1}, q^{c a}\right) \vartheta_{3}\left(w_{1}, q^{a b}\right)=\sum_{\mu, \nu=0}^{\Delta-1} q^{\delta\left(\mu^{2}+b c \nu^{2}\right)} e^{2(\delta \mu x+\delta \nu \nu+\rho z+\tau w) i} \\
\cdot \vartheta_{3}\left(\Delta x-\delta \mu k i, q^{\Delta}\right) \vartheta_{3}\left(\Delta y-b c \delta \nu k i, q^{b c \Delta}\right) \vartheta_{3}\left(\Delta z-c a \rho k i, q^{c a \Delta}\right) \vartheta_{3}\left(\Delta w-a b \tau k i, q^{a b \Delta}\right) ;
\end{gathered}
$$




$$
\begin{aligned}
x_{1} & =s x-t y-u z-v w \\
y_{1} & =b c t x+s y+b v z-c u w \\
z_{1} & =c a u x-a v y+s z+c t w, \\
w_{1} & =a b v x+a u y-b t z+s w \\
\rho & =-A \mu+b B \nu \\
\tau & =-B \mu-C A \nu .
\end{aligned}
$$

10. An extension of Theorem 2. Let $a, b, c$ be positive integers and let $s, t, u, v$ be integers such that

$$
s^{2}+b c t^{2}+c a u^{2}+a b v^{2}=\Delta^{2},
$$

where $\Delta$ is a positive integer prime to $\delta=s^{2}+b c t^{2}$. Let $A, B, \rho, \tau$ be defined as in the preceding paragraph and let

$$
A_{1}=s u+b t v, \quad B_{1}=s v-c t u .
$$

Let $S$ be the totality of quaternions in the form

$$
\Omega=\xi+\eta I+\zeta J+\lambda K,
$$

where $\xi, \eta, \zeta, \lambda$ are integers which satisfy (10). Let $S_{1}$ be the totality of quaternions in the form

$$
\Omega_{1}=\xi_{1}+\eta_{1} I+\zeta_{1} J+\lambda_{1} K,
$$

where $\xi_{1}, \eta_{1}, \zeta_{1}, \lambda_{1}$ are integers such that

$$
\begin{aligned}
& \delta \xi_{1} \equiv-a c A_{1} \zeta_{1}-a b B_{1} \lambda_{1}, \\
& \delta \eta_{1} \equiv a B_{1} \zeta_{1}-a A_{1} \lambda_{1}
\end{aligned}
$$

As in the preceding paragraph, if $\xi, \eta, \zeta, \lambda$ satisfy (10), there is a uniquely determined pair of integers $\mu, \nu ; 0 \leqq \mu<\Delta, 0 \leqq \nu<\Delta$, such that

$$
\xi \equiv \delta \mu, \quad \eta \equiv \delta \nu, \quad \zeta \equiv \rho, \quad \eta \equiv \tau
$$

If we set

$$
\begin{aligned}
\Phi_{\mu \nu} \equiv & q^{\delta\left(\mu^{2}+b c \nu^{2}\right) \Delta^{2}} e^{2(\delta \mu x+\delta b c \nu y+c a \rho z+a b r w) i} \vartheta_{3}\left(\Delta x-\delta \mu \Delta k i, q^{\Delta^{2}}\right) \\
& \cdot \vartheta_{3}\left(b c \Delta y-\delta \nu b c \Delta k i, q^{b c \Delta^{2}}\right) \vartheta_{3}\left(c a \Delta z-\rho c a \Delta k i, q^{c a \Delta^{2}}\right) \vartheta_{3}\left(a b \Delta w-\tau a b \Delta k i, q^{a b \Delta^{2}}\right),
\end{aligned}
$$

it may be shown that

$$
\Phi_{\mu \nu}=\sum q^{\xi^{2}+b c \eta^{2}+c a \zeta^{2}+a b \lambda^{2}} e^{2(\xi x+b c \eta \nu+c a \zeta z+a b \lambda w) i},
$$

where the summation extends over all quadruples which satisfy (15). Hence

$$
\Phi(x, y, z, w) \equiv \sum_{\mu, \nu=0}^{\Delta-1} \Phi_{\mu \nu}=\sum_{\Omega} q^{N(\Omega)} e^{2 i R\left(\Omega X^{\prime}\right)},
$$




\section{T. LATIMER}

where the summation extends over all the elements of $S$ and $X=x+I$ $+z J+w K$.

Set

$$
\rho_{1}=A_{1} \mu-b B_{1} \nu, \quad \tau_{1}=B_{1} \mu+c A_{1} \nu .
$$

In $\Phi_{\mu \nu}$ replace $\rho, \tau$ by $\rho_{1}, \tau_{1}$ respectively and let $\Psi_{\mu \nu}$ be the resulting function. It may be shown as for $\Phi$ that

$$
\Psi\left(x_{1}, y_{1}, z_{1}, w_{1}\right)=\sum_{\mu, \nu=0}^{\Delta-1} \Psi_{\mu \nu}=\sum_{\Omega_{1}} q^{N\left(\Omega_{1}\right)} e^{2 i R\left(\Omega_{1} X_{1}\right)},
$$

where the summation extends over all the elements of $S_{1}$ and $X_{1}=x_{1}+y_{1} I$ $+z_{1} J+w_{1} K$. If we set $\Delta X_{1}=X U, \Delta \Omega_{1}=\Omega U$ where $U=s+t I+u J+w K$, it may be shown that $\Omega_{1}$ belongs to $S_{1}$ if and only if $\Omega$ belongs to $S$ and that the corresponding terms of $\Phi$ and $\Psi$ are equal. Hence

$$
\Phi(x, y, z, w)=\Psi\left(x_{1}, y_{1}, z_{1}, w_{1}\right) .
$$

If we express this in terms of theta functions, replace $q^{\Delta^{2}}, \Delta x, \Delta b c y$, $\Delta c a z, \Delta a b w$ by $q, x, y, z, w$ respectively and make similar replacements for $x_{1}, y_{1}, z_{1}, w_{1}$, we obtain the identity below.

Theorem 4. Let $a, b, c$ be positive integers and let $s, t, u, v$ be integers such that

$$
s^{2}+b c t^{2}+c a u^{2}+a b v^{2}=\Delta^{2},
$$

where $\Delta$ is a positive integer prime to $\delta \equiv s^{2}+b c t^{2}$. Let

$$
\begin{aligned}
& A=s u-b t v, \quad A_{1}=s u+b t v, \quad \rho=-A \mu+b B \nu, \quad \rho_{1}=A_{1} \mu-b B_{1} \nu, \\
& B=s v+c t u, \quad B_{1}=s v-c t u, \quad \tau=-B \mu-c A \nu, \quad \tau_{1}=B_{1} \mu+c A_{1} \nu,
\end{aligned}
$$

where $\mu, \nu$ are arbitrarily chosen integers. Let

$$
\begin{aligned}
F(x, y, z, w) \equiv & \sum_{\mu, v=0}^{\Delta-1} q^{\delta\left(\mu^{2}+b c \nu^{2}\right)} e^{2(\delta \mu x+\delta \nu \nu+\rho z+r w) i / \Delta \vartheta_{3}}\left(x-\frac{\delta \mu k i}{\Delta}, q\right) \\
& \cdot \vartheta_{3}\left(y-\frac{\delta b c \nu k i}{\Delta}, q^{b c}\right) \vartheta_{3}\left(z-\frac{\rho c a k i}{\Delta}, q^{c a}\right) \vartheta_{3}\left(w-\frac{\tau a b k i}{\Delta}, q^{a b}\right),
\end{aligned}
$$

and let $F_{1}$ be the function obtained from $F$ by replacing $\rho, \tau$ by $\rho_{1}, \tau_{1}$ respectively. Then

where

$$
F(x, y, z, w)=F_{1}\left(x_{1}, y_{1}, z_{1}, w_{1}\right)
$$

$$
\begin{array}{ll}
x_{1}=(s x-t y-u z-v w) / \Delta, & z_{1}=(c a u x-a v y+s z+c t w) / \Delta, \\
y_{1}=(b c t x+s y+b v z-c u w) / \Delta, & w_{1}=(a b v x+a u y-b t z+s w) / \Delta .
\end{array}
$$

UNIVERSTTY OF KENTUCKY,

LEXINGTON, $\mathbf{K Y}$. 